\title{
Note on Hypergraphs and Sphere Orders
}

Alexander Schrijver

CWI

THE NETHERLANDS

AND DEPARTMENT OF MATHEMATICS

UNIVERSITY OF AMSTERDAM

AMSTERDAM, THE NETHERLANDS

\section{ABSTRACT}

We show that each partial order $\leq$ of height 2 can be represented by spheres in Euclidean space, where inclusion represents $\leq$. If each element has at most $k$ elements under it, we can do this in $2 k-1$-dimensional space. This extends a result (and a method) of Scheinerman for the case $k=2$. ( 1993 John Wiley \& Sons, Inc.

A partial order $\leq$ on a set $P$ is called a sphere order (in dimension $n$ ) if for each $u \in P$ there exists a ball $B_{u}$ in $\mathbb{R}^{n}$ so that for all $u, v \in P$ one has $u<v$ if and only if $B_{u} \subset B_{v}$. Sphere orders were introduced by Brightwell and Winkler [1], who posed the intriguing question of whether each partially order is a sphere order. They conjectured that the answer is negative.

In [3], Scheinerman showed that each partial order on the vertices and edges of a graph (ordered by inclusion) is a sphere order in dimension 3. Here we extend Scheinerman's result (and his construction) to hypergraphs:

Theorem. For any hypergraph $H=(V, E)$, the partial order on $V \cup E$, given by

$$
x<y \Leftrightarrow x \in V, y \in E, x \in y,
$$

is a sphere order in dimension $2 k-1$, where $k$ is the maximum edge size of $H$.

Since the reverse order to a sphere order is a sphere order again, in the same dimension, we could equally take for $k$ the maximum degree of $H$.

Another formulation of the theorem is that each partial order $P$ of height 2 is a sphere order in dimension $2 k-1$, where $k:=$ $\max _{u \in P}|\{v \in P \mid v<u\}|$.

Journal of Graph Theory, Vol. 17, No. 2, 173-176 (1993)

(C) 1993 John Wiley \& Sons, Inc.

CCC 0364-9024/93/020173-04 
The theorem follows directly from the following lemma (extending the lemma in [3]). Let $C$ be the following curve in $\mathbb{R}^{2 k}$ :

$$
C:=\left\{\left(1, x, x^{2}, x^{3}, \ldots, x^{2 k-1}\right) \mid x \in \mathbb{R}\right\}
$$

Lemma. For each subset $A$ of $C$ with $|A|=k$ there exists a ball $B$ with $B \cap C=A$.

Proof. Let $A$ consist of the points

$$
\left(1, a_{i}, a_{i}^{2}, a_{i}^{3}, \ldots, a_{i}^{2 k-1}\right)
$$

on $C$, for $i=1, \ldots, k$. Let the polynomials $p(x)$ and $q(x)$ be given by

$$
\begin{aligned}
& p(x):=1+x^{2}+x^{4}+\cdots+x^{4 k-2}, \\
& q(x):=\prod_{i=1}^{k}\left(x-a_{i}\right)^{2} .
\end{aligned}
$$

Since $q(x)$ has degree $2 k$, there exists a polynomial $f(x)$ so that the polynomial

$$
r(x):=p(x)-f(x) \cdot q(x)
$$

has degree at most $2 k-1$ (as we can reduce $p(x)$ modulo $q(x)$ to a polynomial of degree at most $2 k-1)$.

Write $r(x)=r_{0}+r_{1} x+r_{2} x^{2}+\cdots+r_{2 k-1} x^{2 k-1}$, and let $g:=\frac{1}{2}\left(r_{0}\right.$, $\left.r_{1}, r_{2}, \ldots, r_{2 k-1}\right)$. Then the ball $B(g,\|g\|)$ with center $g$ and radius $\|g\|$ intersects $C$ exactly in the set $A$. This can be seen as follows.

Let $z=\left(1, x, x^{2}, \ldots, x^{2 k-1}\right)$ be a point on $C$. Then

$$
\begin{aligned}
\|g-z\|^{2} & =\|g\|^{2}+\|z\|^{2}-2 g^{T} z=\|g\|^{2}+p(x)-r(x) \\
& =\|g\|^{2}+f(x) \cdot q(x) .
\end{aligned}
$$

Now the polynomial $f(x)$ has no real zeros, since the polynomial $h(x):=$ $f(x) \cdot q(x)$ has at most $2 k$ real zeros (counting multiplicities). This follows from the fact that the $2 k$ th derivative $h^{(2 k)}(x)$ of $h(x)$ has no real zeros, as it satisfies

$$
\begin{aligned}
h^{(2 k)}(x)= & (2 k) !+\frac{(2 k+2) !}{2 !} x^{2}+\frac{(2 k+4) !}{4 !} x^{4} \\
& +\cdots \frac{(4 k-2) !}{(2 k-2) !} x^{2 k-2}
\end{aligned}
$$

(since $\left.h(x)=p(x)-r(x)=\cdots+x^{2 k}+\cdots+x^{4 k-4}+x^{4 k-2}\right)$. 
As the main coefficient of $f(x)$ is 1 , we know that $f(x)>0$ for all $x \in \mathbb{R}$. So $\|g-z\|^{2}=\|g\|^{2}$ if $z \in A$ and $\|g-z\|^{2}>\|g\|^{2}$ if $z \notin A$.

The theorem now follows by first observing that we may assume that each edge of $H$ contains exactly $k$ vertices (by adding dummy vertices). We take $|V|$ arbitrary points on $C$, to be considered as balls of radius 0 , representing the vertices of $H$. For each edge $e$ of $H$ we take the ball intersecting $C$ exactly in the points representing the vertices in $e$. Since $C$ is in a $2 k-1$-dimensional subspace of $\mathbb{R}$, we obtain a sphere order in dimension $2 k-1$.

We remark that our construction is related to the construction of cyclic polytopes (Gale [2]).

Now one may ask:

Is $2 k-1$ best possible in the theorem (for fixed $k$ )?

We do not know the answer to this question. However, if the balls associated with the vertices of the hypergraph have radius 0 (as is the case in our construction above) then $2 k-1$ is best possible, as follows from the following proposition.

Proposition. There is no subset $V$ of $\mathbb{R}^{2 k-2}$ such that $|V|=2 k+1$ and such that for each subset $X$ of $V$ with $|X|=k$ there exists a ball $B_{X}$ satisfying $B_{X} \cap V=X$.

Proof. Suppose such a set $V$ exists. Then for any two disjoint subsets $X, Y$, of $V$ with $|X|=|Y|=k$ one has that conv $X \cap \operatorname{conv} Y=\varnothing$, since $\operatorname{conv}\left(B_{X} \backslash B_{Y}\right) \cap \operatorname{conv}\left(B_{Y} \backslash B_{X}\right)=\varnothing$.

Let $V=\left\{v_{1}, \ldots, v_{2 k+1}\right\}$. Let $W$ be the linear subspace of $\mathbb{R}^{2 k+1}$ consisting of all vectors $w=\left(w_{1}, \ldots, w_{2 k+1}\right)$ satisfying

$$
\begin{aligned}
w_{1} v_{1}+\cdots+w_{2 k+1} v_{2 k+1} & =0 \\
w_{1}+\cdots+w_{2 k+1} & =0 .
\end{aligned}
$$

Note that $\operatorname{dim} W \geq 2$.

For any vector $w=\left(w_{1}, \ldots, w_{2 k+1}\right)$, let $p_{+}(w)$ be the number of $i \in$ $\{1, \ldots, 2 k+1\}$ satisfying $w_{i}>0$, and let $p_{-}(w)$ be the number of $i \in$ $\{1, \ldots, 2 k+1\}$ satisfying $w_{i}<0$. Now $W$ contains a nonzero vector $w$ satisfying $p_{+}(w) \leq k$ and $p_{-}(w) \leq k$. This can be seen as follows.

Let $W_{+}:=\left\{v \in W \mid p_{+}(v) \geq k+1\right\}$ and $W_{-}:=\left\{v \in W \mid p_{-}(v) \geq k+\right.$ 1\}. So $W_{+}$and $W_{-}$are two disjoint open subsets of $W \backslash\{0\}$. Moreover, $W_{+} \neq$ $\backslash W \backslash\{0\}$ and $W_{-} \neq W \backslash\{0\}$, since $W_{-}=-W_{+}$. Hence by the connectedness of $W \backslash\{0\}, W \backslash\{0\} \neq W_{+} \cup W_{-}$, implying that $W \backslash\{0\}$ contains a vector $w$ satisfying $p_{+}(w) \leq k$ and $p_{-}(w) \leq k$. 
We may assume that $w=\left(w_{1}, \ldots, w_{2 k+1}\right)$ satisfies $w_{1}, \ldots, w_{k} \geq 0$, $w_{k+1}, \ldots, w_{2 k} \leq 0, w_{2 k+1}=0$ and $w_{1}+\cdots+w_{k}=1$. Hence $\left(-w_{k+1}\right)+$ $\cdots+\left(-w_{2 k}\right)=1$. In particular, both $\operatorname{conv}\left\{v_{1}, \ldots v_{k}\right\}$ and $\operatorname{conv}\left\{v_{k+1}, \ldots\right.$, $\left.v_{2 k}\right\}$ contain the vector

$$
w_{1} v_{1}+\cdots+w_{k} v_{k}=\left(-w_{k+1}\right) v_{k+1}+\cdots+\left(-w_{2 k}\right) v_{2 k}
$$

This contradicts the fact that $\operatorname{conv}\left\{v_{1}, \ldots v_{k}\right\} \cap \operatorname{conv}\left\{v_{k+1}, \ldots, v_{2 k}\right\}=\varnothing$.

\section{I}

Thus if $|V|=2 k+1$ and $E$ consists of all subsets of $V$ of size $k$, then $2 k-1$ is best possible in the theorem if each ball associated with a vertex in $V$ has radius 0 .

\section{ACKNOWLEDGMENTS}

I am grateful to the referees for helpful comments and suggestions, in particular for suggesting question (8).

\section{References}

[1] Graham Brightwell and Peter Winkler, Sphere orders. Order 6 (1989) 235-240.

[2] David Gale, Neighborly and cyclic polytopes. Convexity, Proceedings of Symposia in Pure Mathematics Volume VII, American Mathematical Society, Providence, RI (1963) 225-232.

[3] Edward R. Scheinerman, A note on graphs and sphere orders. J. Graph Theory. To appear. 